${ }^{1}$ Facultad de Salud, Escuela de Kinesiología, Universidad Santo Tomás, sede Viña del Mar. Viña del Mar, Chile.

${ }^{2}$ Laboratory of Morphological Sciences, Instituto de Biología. Pontificia Universidad Católica de

Valparaíso. Valparaíso, Chile.

${ }^{3}$ Departamento de Deporte e Informática, Universidad Pablo de Olavide. Sevilla, España. aKinesiólogo ${ }^{b}$ Magister. 'PhD.

Trabajo no recibió financiamiento. Los autores declaran no tener conflictos de interés.

Recibido el 10 de septiembre de 2018, aceptado el 10 de enero de 2019.

Correspondencia a: Tuillang Yuing Farías Universidad Santo Tomás. Avda. 1 Norte 3041, Viña del Mar. Región de Valparaíso, Chile. tuillangyuing@santotomas.cl

\section{Hemoglobina glicada y ejercicio: una revisión sistemática}

\author{
TUILLANG YUING ${ }^{1, a, b}$, PABLO A. LIZANA ${ }^{2, b, c,}$ \\ FRANCISCO JOSÉ BERRAL ${ }^{3, b, c}$
}

\section{Effects of physical training in patients with type 2 diabetes mellitus: a systematic review}

Physical training is recommended in several studies and guidelines for the control of type 2 diabetes mellitus (DM2) and its complications. We performed a systematic review about the effects of aerobic training (AT), resistance $(R T)$ or the combination of both (AT/RT), on glycated hemoglobin (HbA1c) in patients with DM2. Therefore, we included 15 clinical trials with at least 12 weeks duration about training program or recommendations of physical exercise, that evaluated the reduction in HbA1c levels in patients with DM2. Information was obtained on training modality ( $A T, R T$ or $A T / R T$ ), training parameters, duration and weekly training frequency. The results showed increases in peak or maximal oxygen uptake, exercise tolerance time and muscle strength, depending on the type of training, and a reduction in $\mathrm{HbAl}$ levels. We conclude that exercise training is associated with reductions of HbA1c in patients with DM2. Thus, it can be a complementary tool in the management of these patients.

(Rev Med Chile 2019; 147: 480-489)

Key words: Diabetes Mellitus, Type 2; Exercise; Glycated Hemoglobin A.
L a prevalencia de la DM está aumentando en todo el mundo, lo que resulta en una epidemia mundial ${ }^{1}$. Se estima que para el 2040 , el número total de diabéticos en todo el mundo sería de 642 millones lo que se traduce en un aumento del gasto público en salud y una sobrecarga económica ${ }^{2}$. El tipo más común, la diabetes mellitus tipo 2 (DM 2), representa el 90-95\% de los casos y se caracteriza por una alteración en su acción y/o en la secreción de insulina. Esta enfermedad es más frecuente en personas mayores de 45 años de edad y con algún grado de sobrepeso ${ }^{3}$. En Chile, habría 1.372.700 personas con DM2, lo que representa el 11\% de la población adulta entre 20-79 años. Esta cifra colocaría a nuestro país en el segundo lugar de sudamérica, situación que se asemeja a la observada en la frecuencia de trastornos nutricionales por exceso ${ }^{4}$. Sin embargo, como resultado del aumento del sobrepeso y obesidad a menores edades, la DM2 está aumentando entre niños y adultos jóvenes ${ }^{5}$. La creciente prevalencia de DM2 también se puede atribuir a otros factores, como el crecimiento de la población y el envejecimiento, cambio en los estilos de vida, la creciente prevalencia de la obesidad y la inactividad física, así como una mayor supervivencia entre los pacientes con $\mathrm{DM} 2^{3}$. De los factores mencionados hasta ahora, el factor de riesgo más importante para la regulación de la concentración plasmática de glucosa, sería la inactividad física muscular, ya que los músculos esqueléticos son los principales encargados de la captación y utilización de la glucosa, llegando a consumir hasta 80 a $90 \%{ }^{6}$.

El entrenamiento físico corresponde a un elemento esencial en el manejo de la diabetes en conjunto con el tratamiento farmacológico y modificaciones del estilo de vida ${ }^{1}$ por lo que ha sido considerado como uno de los tres componentes principales en el control y tratamiento de la $\mathrm{DM} 2^{8}$. Es característico que pacientes con DM2 tengan 
menor capacidad aeróbica, niveles más bajos de fuerza muscular y flexibilidad cuando se comparan con individuos sanos de la misma edad y sexo. Las personas diabéticas que son físicamente activas y/o presentan una buena capacidad aeróbica presentan un mejor pronóstico que aquellos que son inactivos y/o aquellos con baja capacidad aeróbica ${ }^{8}$. Las directrices actuales de la Asociación Americana de Diabetes (ADA) ${ }^{9}$, recomiendan realizar EA de $\geq 150$ min de intensidad moderada o $\geq 90 \mathrm{~min}$ de intensidad vigorosa por semana $\mathrm{y} / \mathrm{o}$ $E R \geq 3$ veces/semana, teniendo mayor adherencia a los protocolos de EA que de $\mathrm{ER}^{10}$. Un estudio reciente ${ }^{11}$, ha reportado que el entrenamiento de intensidad moderada a vigorosa genera cambios significativos en personas diabéticas, mejorando el control glicémico y otorgando una importancia clave a la intensidad del entrenamiento.

Por lo visto hasta ahora, tanto el entrenamiento aeróbico como los de fuerza pueden mejorar o aumentar la acción insulina, controlar los niveles de glicemia, disminuyendo el riesgo de enfermedades cardiovasculares $^{12}$, reducción de la mortalidad ${ }^{13}$, prevenir complicaciones relacionadas con la DM y mejorar la calidad de vida de los diabéticos cuando se realiza continuamente ${ }^{14}$. Recientemente, un metaanálisis hecho por Davies et al. ${ }^{15}$ mostró una reducción general en $\mathrm{HbAlc}$ de $0,8 \%$ con entrenamientos de resistencia eincremento de 11,8\% en el pico de $\mathrm{VO}_{2}$ con entrenamientos aeróbicos. Estos factores hacen que la inclusión del entrenamiento físico sea un elemento fundamental del tratamiento de la DM2.

Es por esto, que este estudio consiste en una revisión sistemática sobre las asociaciones y cambios que produciría el EA, ER o el EA/ER sobre el control glicémico $y$, aportar conocimiento a los profesionales de la salud que trabajan con personas con diabetes, obesidad o síndrome metabólico.

\section{Métodos}

\section{Estrategia de búsqueda y selección de estudios}

Se buscó en las bases de datos electrónicas MEDLINE (accedido por PubMed), EMBASE, RESEARCH GATE entre el período de noviembre de 2008 y agosto de 2017. Adicionalmente, buscamos las referencias de estudios publicados manualmente. La búsqueda inicial comprendió los términos MeSH: \{Glycated hemoglobin A, type 2 diabetes \& physical activity\} o su correspondientes en español: \{Hemoglobina glicada A, diabetes tipo 2 y actividad física $\}$ y asociados al término boleano $\{\mathrm{AND}\} /\{\mathrm{y}\}$ respectivamente. Solo textos completos elegibles en inglés o español fueron considerados para la revisión. Esta revisión sistemática está descrita de acuerdo a los criterios de elementos de informes preferidos para revisiones sistemáticas y metaanálisis (PRISMA) ${ }^{16}$. El flujograma de búsqueda se muestra en la Figura 1.

\section{Criterio de elegibilidad}

Se incluyeron ensayos clínicos que compararon entrenamientos programados (EA, ER o EA/ER) $o$ directrices de actividad física en pacientes con DM2 mayores de 18 años de ambos sexos, que evaluó la HbA1c como un resultado del entrenamiento de a lo menos 12 semanas de entrenamiento, ya sea como valores promedio, diferencias de promedio y valores de dispersión respectivos de HbAlc al inicio y después de la intervención.

Los programas de entrenamiento fueron definidos como una intervención en pacientes en que el programa de entrenamiento fuera individualizado y supervisado. Las recomendaciones o directrices de actividad física se definieron como una intervención en la cual los pacientes estaban parcialmente o no participando en programas de entrenamiento supervisado, pero habiendo recibido instrucciones formales para ejercitar regularmente con o sin un individuo a cargo de la prescripción del entrenamiento. Los estudios elegibles incluyeron solo individuos capaces de realizar el entrenamiento, sin limitaciones clínicas. Se implementaron los siguientes criterios de inclusión: (i) diseños de estudio longitudinal con al menos dos grupos de intervención (no se consideraron los estudios de efectos agudos o a corto plazo), (ii) intervención física EAo ERpor sí sola y (iii) EA/ER. Los criterios de exclusión fueron los siguientes: (i) estudios de pacientes con diabetes tipo 1 o diabetes gestacional; (ii) estudios en que no le hicieron o no proporcionan información sobre las asociaciones de la intervención con $\mathrm{HbAlc}$ en el grupo experimental, el grupo control, o ambos; (iii) estudios con menos de 12 semanas de intervención.

\section{Extracción de datos}

Se recolectaron los títulos y resúmenes de los artículos seleccionados. Si los resúmenes no apor- 


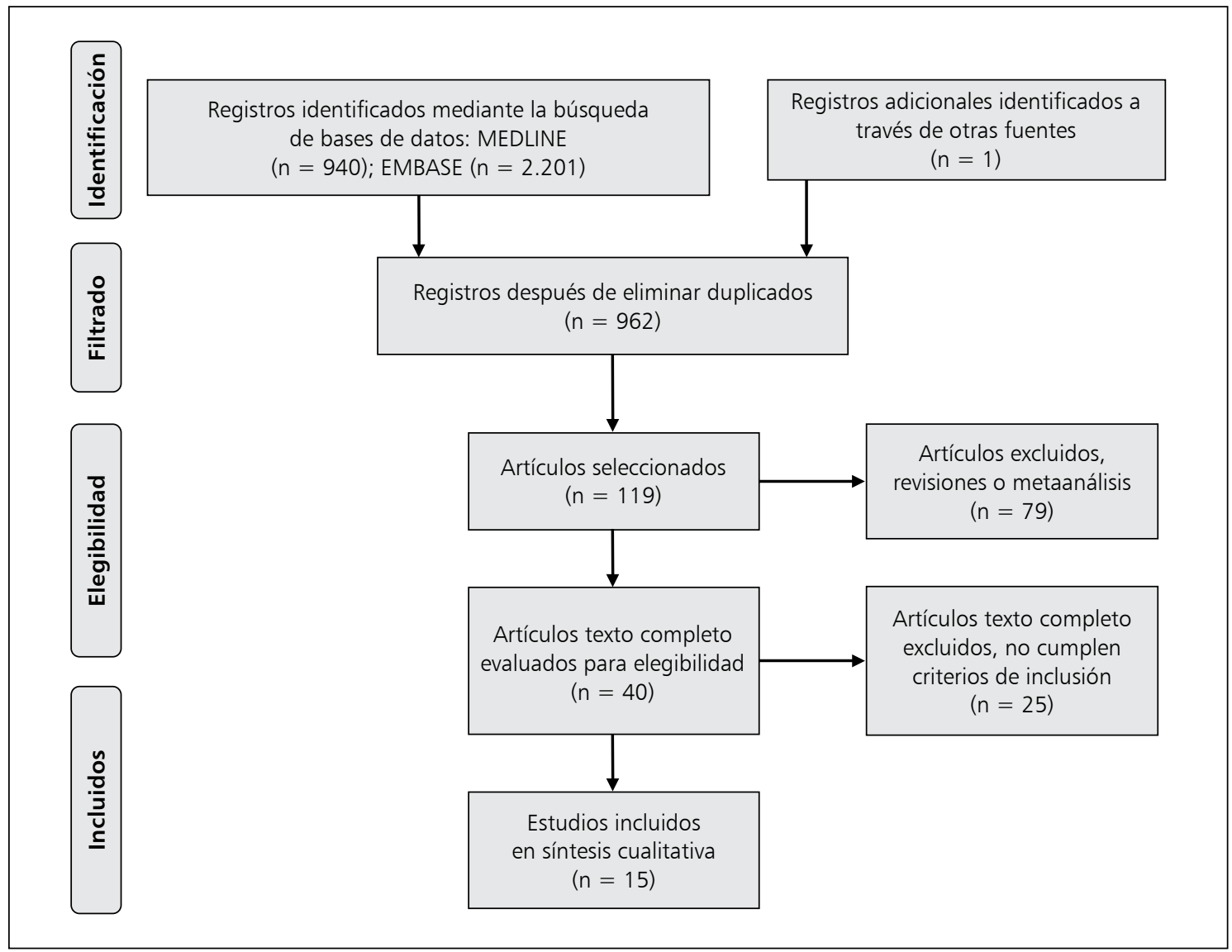

Figura 1. Flujograma de búsqueda de acuerdo a los criterios PRISMA ${ }^{17}$.

taban suficiente información sobre los criterios de inclusión y exclusión, se descargaba el texto completo para su evaluación.

Se ilustra un diagrama de flujo del proceso de selección del estudio en la Figura 1. La búsqueda recuperó un total de 962 artículos relevantes probables de analizar. Todos los artículos recuperados fueron revisados por dos revisores externos (CC y PH). Las decisiones frente a las dudas para inclusión/exclusión fueron resueltos por un tercer revisor (SL). Después de la proyección por título y/o resumen, 79 artículos fueron excluidos debido a (i) irrelevancia del tema, (ii) siendo metanálisis/documentos de revisión, (iii) no inclusión de $\mathrm{HbAlc}$ como valoración de resultados y (iv) la inclusión de una o más comorbilidades. Los 40 artículos restantes se evaluaron como potencialmente documentos pertinentes y los documentos completos fueron adquiridos. Después de examinar los artículos completos, 25 artículos fueron excluidos por las siguientes razones: períodos de intervención $<12$ semanas, pruebas de control glicémico diferentes, o un diseño de estudio transversal o sin intervención de entrenamiento físico, o ausencia de evaluaciones cognitivas o motoras en línea base o punto final. Los artículos restantes $(n=15)$ cumplieron todos los criterios de inclusión y se incluyeron en la revisión.

\section{Resultados}

Las características de todos los estudios analizados se detallan en la Tabla 1.

Uno de los primeros artículos analizados corresponde al publicado en el año 2008 por el grupo liderado por Marcus ${ }^{17}$, en el cual partici- 


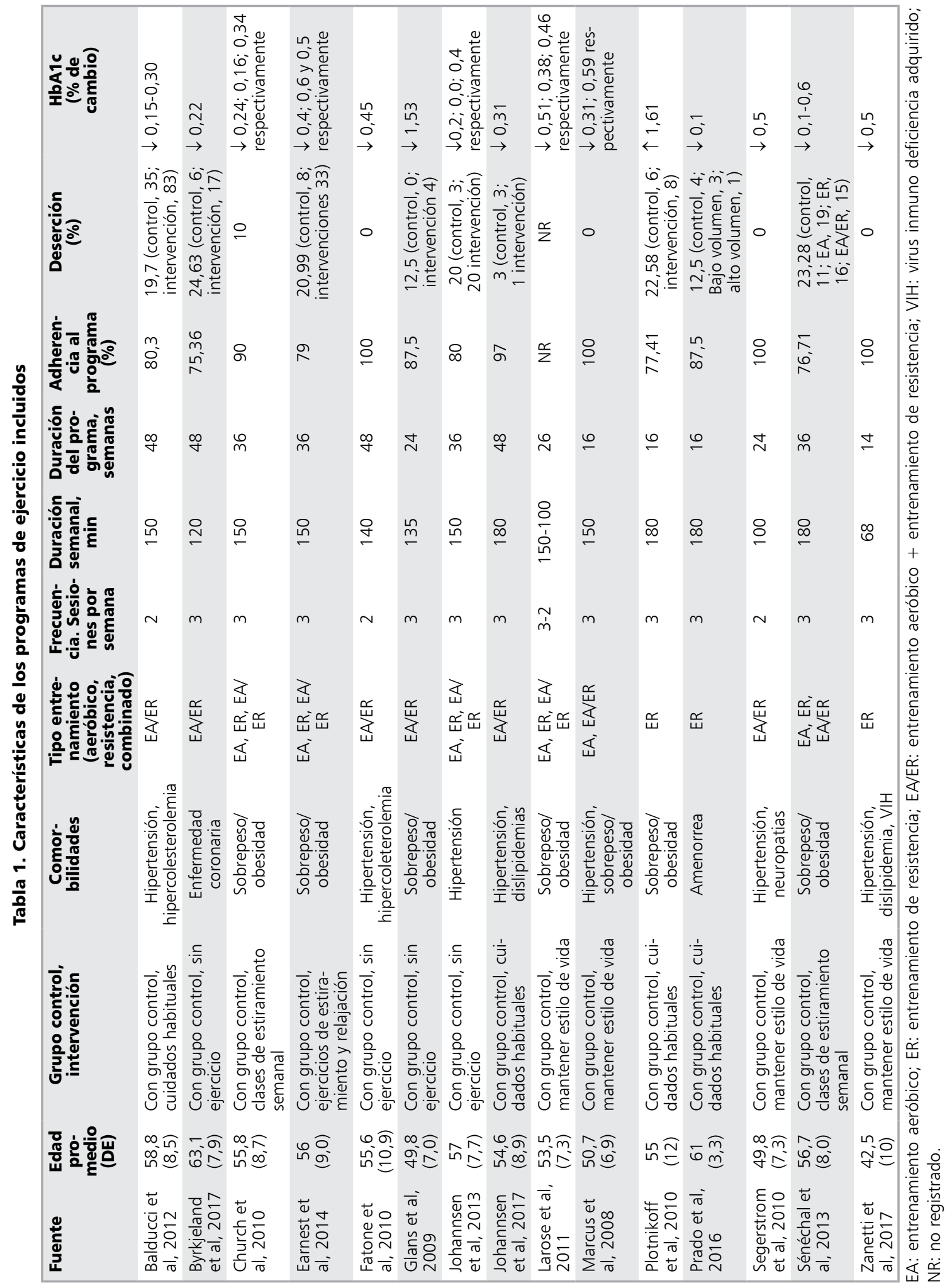


paron un total de 22 pacientes con DM2 y que fueron aleatoriamente distribuidos para realizar dos modalidades de entrenamiento(aeróbico de intensidad moderada a $60 \%$ de intensidad (EA) y aeróbico de intensidad moderada adicionando la realización de entrenamientode resistencia en prensa de pierna(EA/ER)). Un total de 15 pacientes completaron el programa de entrenamiento supervisado de 16 semanas. Ambos grupos de entrenamiento completaban semanalmente $150 \mathrm{~min}$ de ejercicio. No hubo diferencias significativas entre los grupos con respecto al cambio en las mediciones de $\mathrm{HbAlc}$. Si hubo un efecto principal significativo para el tiempo $(p=0,02)$. La media de cambio para la HbAlc fue de 0,59\% (IC 95\% = -1,5 a 0,28 ) para el grupo EA/ER y $-0,31 \%$ (IC $95 \%=-0,60$ a -0,03) para el grupo AE.

Posteriormente, con un protocolo EA/ER, Glans $(2009)^{18}$, demostró un incremento en la sensibilidad de la insulina $\left(2,7 \pm 1,4 \mathrm{mg} \mathrm{kg}^{-1} \mathrm{~min}^{-1}\right.$ vs $\left.3,4 \pm 2 \mathrm{mg} \mathrm{kg}^{-1} \mathrm{~min}^{-1}, \mathrm{p}<0,05\right)$ en todos los pacientes, con una mejora notable en la $\mathrm{HbAlc}(6,5$ $\pm 1,1$ vs $6,3 \pm 1,0 \mathrm{p}<0,05)$ y sin modificaciones significativas en el perfil lipídico.

De los estudios publicados recientemente, es interesante destacar el trabajo de Byrkjeland ${ }^{19} \mathrm{y}$ el de Zanetti ${ }^{20}$, ya que en el caso del primero a pesar de haber reportado en estudios previos cambios no significativos en el porcentaje de $\mathrm{HbAlc}$, en un estudio posterior reportó cambios significativos con un entrenamiento EA/ER, en donde estuvo asociado a cambios en la función endotelial como la E-selectina $(\mathrm{r}=0,56, \mathrm{p}<0,001)$, la molécula de adhesión celular-I $(r=0,27, p=0,052)$, entre otras; pero sin una mejora significativa en la función endotelial en pacientes con enfermedad coronaria. El estudio de Zanetti en cambio, estudió el efecto de un programa de entrenamiento de resistencia no lineal (NLRS) en un grupo de sujetos con VIH y comprobó que este tipo de ejercicio disminuía la posibilidad de desarrollar los componentes del síndrome metabólico al obtener una disminución significativa de lamasa de grasa $(-10,1 \%, \mathrm{p}<0,0001)$, porcentaje de grasa corporal $(-11,4 \%, \mathrm{p}<0,0001)$, perímetro de la cintura $(-1 \%, \mathrm{p}=0,001)$, glucosa en ayunas $(-13,7 \%, \mathrm{p}=0,012)$ y HbA1c $(-9 \%, \mathrm{p}<0,0001)$ y aumentando la masa magra $(+5,5 \%, \mathrm{p}<0,0001)$ y los niveles de lipoproteínas de alta densidad (HDL) $(+31 \%, \mathrm{p}<0,0001)$.

\section{Precauciones/Seguridad del entrenamiento}

En todos los ensayos clínicos revisados los sujetos fueron médicamente autorizados para realizar el programa de entrenamiento. Si era necesario, se realizaba una prueba de esfuerzo antes de iniciar el entrenamiento para garantizar la autorización para la participación ${ }^{17,21}$. Todos los sujetos tenían cognición normal. Se excluían de participar sujetos con enfermedad cardíaca severa; hipertensión no controlada; problemas ortopédicos que limitaron su capacidad de realizar los entrenamientos sin dolor; trastornos del sistema nervioso central o periférico; retinopatía diabética; miopatía; enfermedad reumatológica que afecta la movilidad; con el fin de minimizar los riesgos ${ }^{17,18,19,20}$. Del mismo modo la frecuencia cardíaca era monitoreada antes, durante y posterior al ejercicio con un monitor de frecuencia cardíaca. Otra medida de control, corresponde al registro de la percepción subjetiva de esfuerzo a través de la escala de Borg ${ }^{18}$. Todos los programas de entrenamiento fueron supervisados por personal especializado, lo que permite un completo control de las sesiones de entrenamiento y además que la mayoría de los participantes cumplan con un mayor número de sesiones, mejorando la adherencia y reduciendo la deserción. Estos datos se muestran en la Tabla 1.

Los efectos adversos son relativamente escasos cuando se cuenta con supervisión constante y se toman las medidas de monitoreo cardíaco y glicémico, por lo que el registro previo a las sesiones de entrenamiento de presión arterial (PA), frecuencia cardíaca y glicemia se hacen necesarios. En el reporte de Marcus y colaboradores ${ }^{17}$ se hace una detallada descripción de los cuidados previos, así como también las normas para detener la sesión de ejercicio. Dentro de estas medidas se destacan: Una PA previa al ejercicio $<140 / 90 \mathrm{mmHg}$ para que los sujetos comenzaran el ejercicio. Si la PA antes de comenzar era mayor a ese valor, se sentaba al sujeto durante 10 min y se reevaluaba. $\mathrm{Si}$ algún participante presentaba una glicemia $<a$ $100 \mathrm{mg} / \mathrm{dl}$ antes de comenzar se le indicaba un refrigerio de $15 \mathrm{~g}$ de carbohidratos y volver a evaluar los niveles de glucosa en sangre para minimizar el riesgo de hipoglicemia. Si el nivel de glucosa en sangre antes del ejercicio era $>300 \mathrm{mg} / \mathrm{dl}$, los sujetos comenzaron a hacer ejercicio y se volvían a evaluar en 20 a 30 min para asegurarse de que el nivel de glucosa en sangre no aumentara. Si el 
nivel de glucosa en sangre estaba aumentando, el ejercicio se detenía.

Por último, una de las medidas que permite controlar los efectos de los programas de entrenamiento, es contar con elapoyo telefónico para favorecer la adherencia a los programas ${ }^{17,19,20,22}$.

\section{Protocolos de entrenamiento}

Si bien no existe un consenso con respecto a la modalidad de entrenamiento físico más adecuado para el abordaje de la DM2, si existe una similitud y constancia de parámetros para la prescripción. En la Tabla 2 se resumen los parámetros asociados a la dosimetría de los tipos de entrenamiento y los resultados principales obtenidos.

Entrenamiento aeróbico (EA): incluye modalidades de ejercicios rítmicos que involucran grandes grupos musculares para mejorar la resistencia cardiovascular, incluyendo una etapa de calentamiento, una fase principal y una de vuelta a la calma, realizando de 2 a 3 sesiones semanales a una intensidad entre 50 a $80 \%$ del $\mathrm{VO}_{2}$ máx, o su equivalente en porcentaje de la frecuencia cardíaca de reserva y que permita completar $150 \mathrm{~min}$ de entrenamiento semanal, o, completar un gasto energético de $12 \mathrm{kcal} / \mathrm{kg}$ de peso corporal por semana $^{22-25}$.

Dada la labilidad de los sistemas cardio respiratorio y la alteración de la regulación metabólica frente a la realización de esfuerzo físico que implica una sobrecarga a estos sistemas, es que es deseable la continua supervisión y monitoreo de parámetros cardíacos (frecuencia cardíaca y PA), ventilatorios $\left(\mathrm{VO}_{2}\right.$, frecuencia ventilatoria, saturación de oxígeno), antes, durante y al finalizar las sesiones de entrenamiento ${ }^{18,23,24,26}$.

De la misma manera, se definen dosimetrías de entrenamiento de moderada a alta intensidad, $y$, que a medida que los participantes se vayan adaptando a las cargas de trabajo, estas se vayan incrementando de acuerdo a la tolerancia y capacidad de cada sujeto.

Entrenamiento de resistencia (ER): en los últimos años se ha observado como paulatinamente la evidencia científica le ha atribuido cada vez más importancia al parámetro de intensidad del esfuerzo y a su vez los beneficios que trae el ER en pacientes DM2. En la gran mayoría de los estudios analizados, se realizan de 2 a 3 sesiones semanales ${ }^{27-29}$, desarrollando un trabajo de 4 grupos musculares de la mitad superior del cuerpo en el sentido transversal, donde se incluyen press banca, remo sentado, jalón posterior y press de hombro) y 3 ejercicios para la mitad inferior del cuerpo, incluyendo: prensa de piernas, extensión de piernas y curl de femorales) y 2 ejercicios para abdominales o espalda baja, con intensidades que fluctúan entre 60 a $80 \%$ de 1 RM (1 repetición máxima), o que permita realizar entre 10 a 12 repeticiones por serie ${ }^{27,29,30,31}$.

Entrenamiento combinado (EA/ER): para este tipo de entrenamiento se seleccionaba un EA que implicara un gasto energético semanal de $10 \mathrm{kcal} /$ $\mathrm{kg}$ de peso corporal por semana ${ }^{22,24,25}$, sumado a 2 sesiones de ER, realizando sólo 1 serie de 10 a 12 repeticiones para el total de grupos musculares indicados, para asegurar una equivalencia en tiempo con los EA y ER $20,22,24,25$.

Finalmente, cabe destacar que la gran mayoría de los estudios analizados, demostraron una reducción en los niveles de HbAlc en todos los grupos de entrenamiento, sin encontrar mayores diferencias entre ellos, y que estas reducciones se daban asociadas o no a cambios en la masa magra o en la masa grasa, pero que si estaban asociados en todos los casos a un aumento en la capacidad funcional de los sujetos y/o de la fuerza muscular de los grupos musculares de las mitades superior e inferior del cuerpo ${ }^{17,21,26,30}$.

\section{Conclusión}

Las investigaciones demuestran mejoras significativas en variados parámetros indicadores de control glicémico, especialmente la HbAlc, con la intervención de programas de EA y ER por si solos, como así también EA/ER, no generando diferencias entre los diferentes entrenamientos, pero sí todos ellos teniendo una característica común que es un incremento en la capacidad física y tolerancia al esfuerzo, como así también en mejoras de la fuerza muscular.

Agradecimientos: A los revisores externos por su colaboración en la selección de los artículos. A la Dirección de Investigación y Post grado de la Universidad Santo Tomás y a la Vice rectoría de investigación de la Pontificia Universidad Católica de Valparaíso por su constante apoyo en el desarrollo de proyectos de investigación y difusión científica. 


\begin{tabular}{|c|c|c|c|c|c|c|c|c|}
\hline 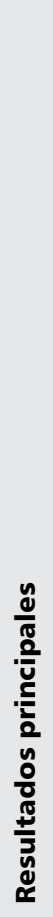 & 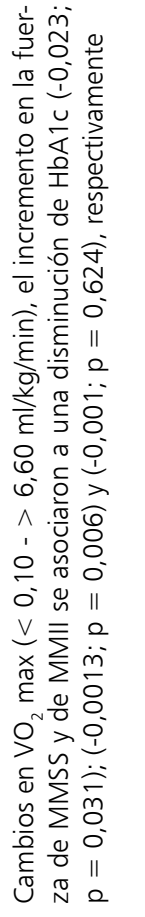 & 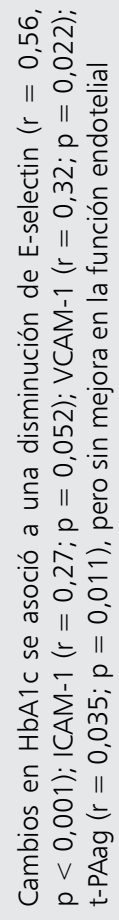 & 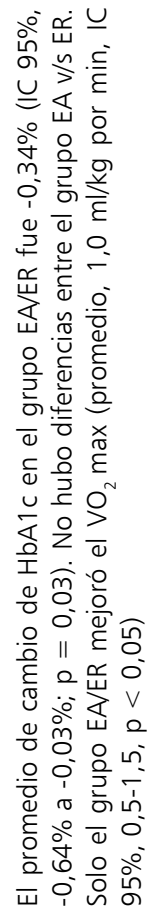 & 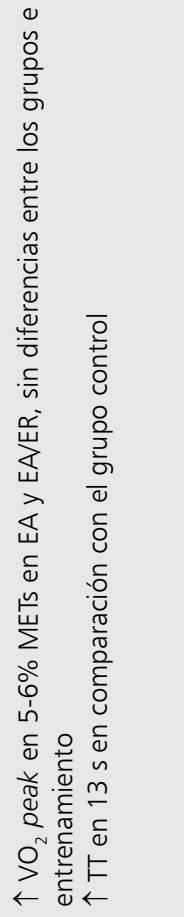 & 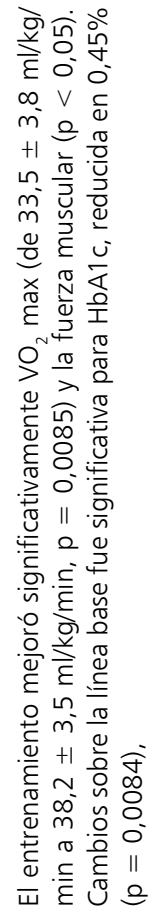 & 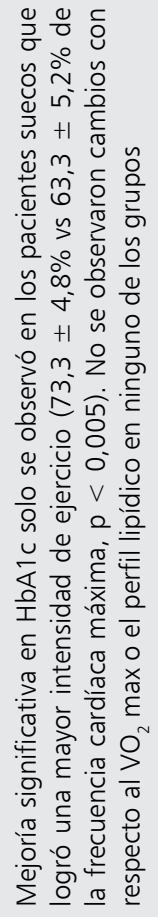 & 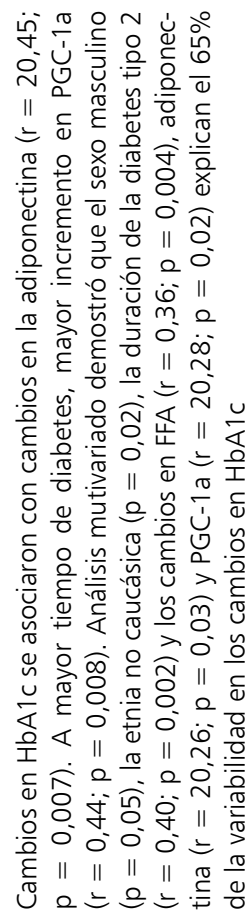 & 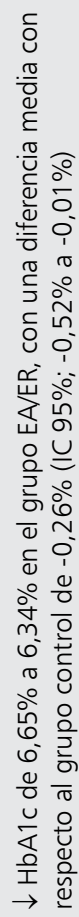 \\
\hline 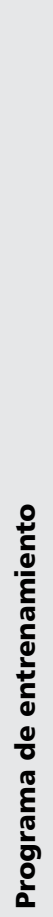 & 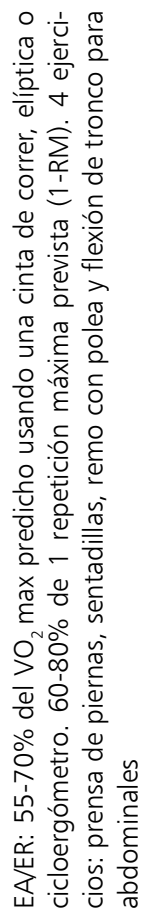 & 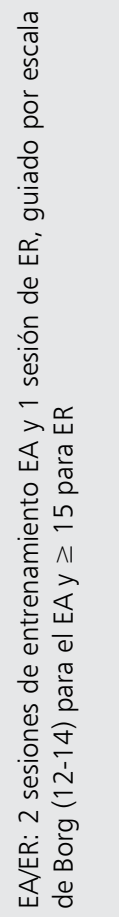 & 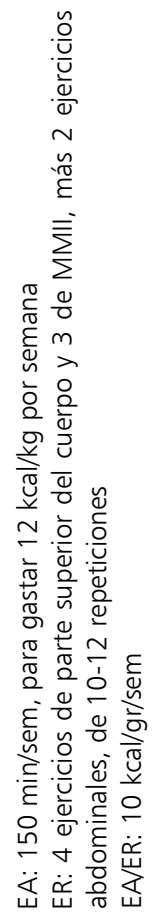 & 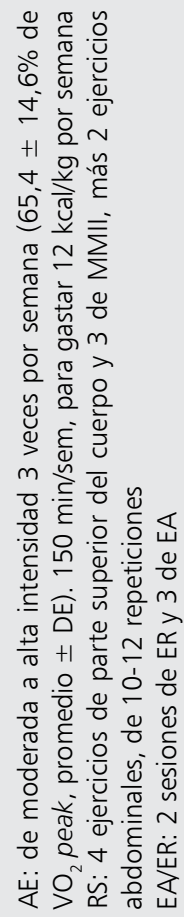 & 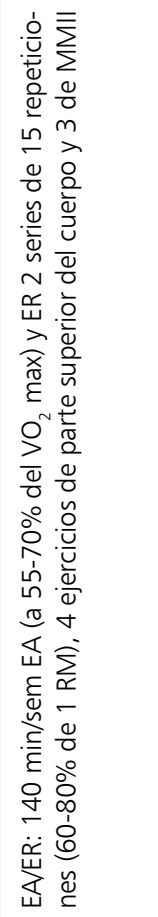 & 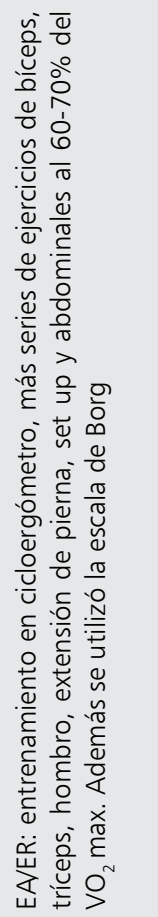 & 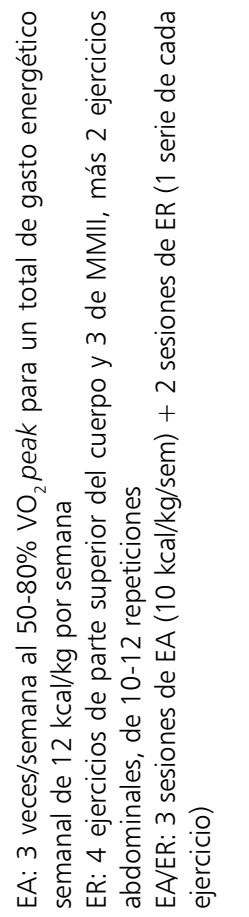 & 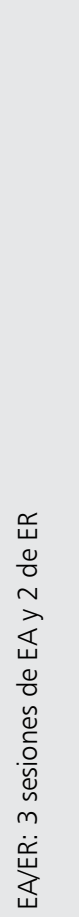 \\
\hline 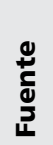 & 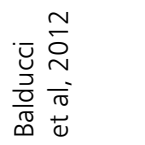 & 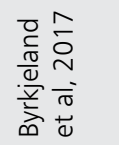 & 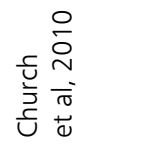 & 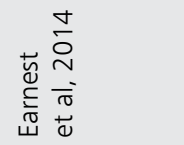 & 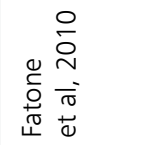 & 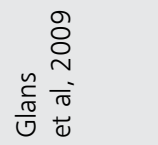 & 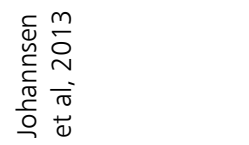 & \\
\hline
\end{tabular}




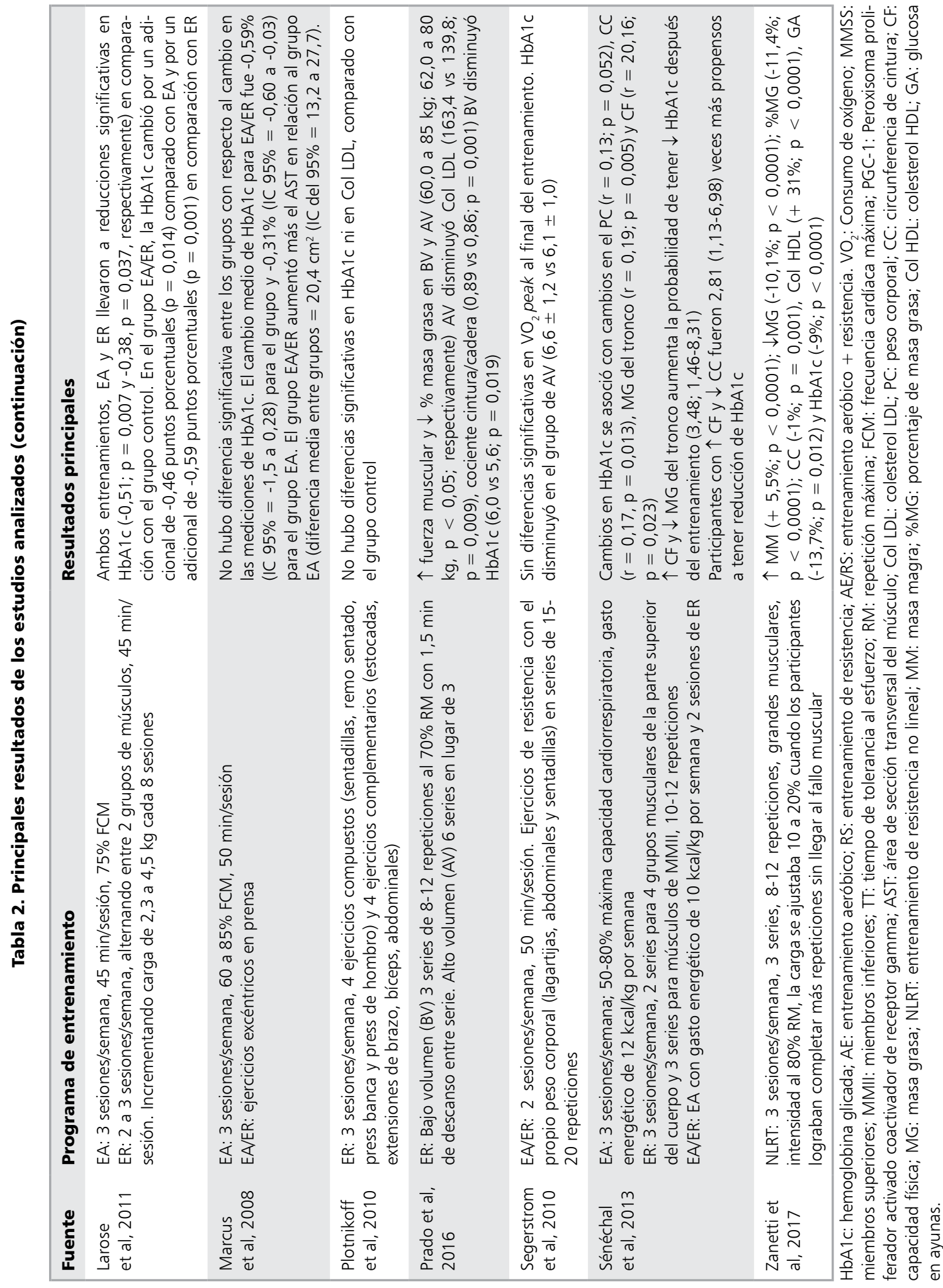




\section{Referencias}

1. de Ladeet al. Effects of different exercise programs and minimal detectable changes in hemoglobin Alc in patients with type 2 diabetes. Diabetol Metab Syndr 2016; (13) 8: 2-9.

2. Cho NH, Shaw JE, Karuranga S, Huang Y, da Rocha Fernández JD, et al. IDF Diabetes Atlas: Global estimates of diabetes prevalence for 2017 and projections for 2045. Diabet Res ClinPrac 2018; 138 (2): 271-281.

3. Lorber D. Importance of cardiovascular disease risk management in patients with type 2 diabetes mellitus. Diabetes Metab Syndr Obes 2014;7 (5): 169-83.

4. Sapunar J. Epidemiología de la diabetes mellitus en Chile. Rev med clin condes 2016; 27 (2): 146-51.

5. Pandey A, Chawla S, Guchhait P. Type-2 Diabetes: $\mathrm{Cu}-$ rrent Understanding and Future Perspectives. Int Un Bioch Mol Bio 2015; 67 (7): 506-13.

6. DeFronzo R, Gunnarsson R, Björkman O, Olsson M and Wharen J. Effects of Insulin on Peripheral and Splanchnic Glucose Metabolism in Noninsulin-dependent (Type 11) Diabetes Mellitus. J Clin Invest 1985; 76 (7): 149-55.

7. Moura B, Natali A, Marins J, Amorim P. Different approaches of physical training used in the management of type 2 diabetes: a brief systematic review of randomized clinical trials. Br J Diabetes Vasc Dis 2011; 11 (9): 210-6.

8. Hoi Yun Yu O, Suissa S. Identifying causes for excess mortality in patients with diabetes: Closer but not there yet. Diabetes Care 2016; 39 (11): 1851-3.

9. American Diabetes AssociationDiabetes Care 2017; 40 (Supplement 1): S11-S24.

10. Mu L, Cohen A, and Mukamal K. Resistance and aerobic exercise among adults with diabetes in the U.S. Diabetes Care 2014; 37: 175-6.

11. Bakrania K, Yates T, Edwardson C, Bodicoat D, Esliger $\mathrm{D}$, et al. Associations of moderate-to-vigorous intensity physical activity and body mass index with glycated haemoglobin within the general population: a cross-sectional analysis of the 2008 Health Survey for England. BMJ Open 2017; 7 (2): 1-8.

12. Boulé N, Haddad E, Kenny G, Wells G, Sigal R. Effects of exercise on glycemic control and body mass in type 2 diabetes mellitus. JAMA 2001; 286 (10): 1218-27.

13. Sluik D, Buijsse B, Muckelbauer R, Kaaks R, Teucher $\mathrm{B}$, Johnsen $\mathrm{N}$, et al. Physical activity and mortality in individuals with diabetes mellitus. A prospective study and meta-analysis. JAMA 2012; 172 (17): 1285-95.

14. Sigal R, Kenny G, Boulé NG, Wells G, Prud'homme D, Fortier M, et al. Effects of aerobic training, resistance training, orbothon glycemic control in type 2 diabetes. Ann Intern Med 2007; 147 (6): 357-69.

15. Davies M, D’Alessio D, Fradkin J, Kernan W, et al. Management of hyperglycaemia in type 2 diabetes, 2018. A consensus report by the American Diabetes Association (ADA) and the European Association for the Study of Diabetes (EASD). Diabet 2018; 61 (10): 2461-98.

16. Moher D, Liberati A, Tetzlaff J, Altman DG. Preferred Reporting Items for Systematic Reviews and Meta-Analyses: The PRISMA Statement. PLoS Med 2009; $6(6): 1-6$.

17. Marcus R, Smith S, Morell G, Addison O, Dibble L, et al. Comparison of combined aerobic and high-force eccentric resistance exercise with aerobic exercise only for people with type 2 diabetes mellitus. Phys Ther 2008; 88 (11): 1345-54.

18. Glans F, Eriksson KF, Segerstrom A, Thorson O, Wollmer P, et al. Evaluation of the effects of exercise on insulin sensitivity in arabian and swedish women with type 2 diabetes. Diabet Res Clin Pract 2009; 85 (4): 6974.

19. Byrjkeland R, Njerve I, Arnesen H, Seljeflot I, Solheim $\mathrm{S}$. Reduced endothelial activation after exercise is associated with improved $\mathrm{HbAlc}$ in patients with type 2 diabetes and coronary artery disease. Diabet Vasc Dis Res 2017; 14 (2) 94-103.

20. Zanetti H, da Cruz L, Lourenco C, Neves F, ¿Silva-Vergara $\mathrm{M}$, et al. Does nonlinear resistance training reduce metabolic syndrome in people living with HIV?: A randomized clinical trial. J Sports Med Phys Fitness 2017; 57 (5): 678-84.

21. Fatone C, Guescini M, Balducci S, Battistoni S, Settequattrini A, et al. Two weekly sessions of combined aerobic and resistance exercise are sufficient to provide beneficial effects in subjects with type 2 diabetes mellitus and metabolic síndrome. J Endocrinol Invest 2010; 33 (10): 489-95.

22. Church T, Blair S, Cocreham S, Johannsen N, Jhonson $\mathrm{W}$, et al. Effects of aerobic and resistance training on hemoglobin Alc levels in patients with type 2 diabetes: A randomized controlled trial. JAMA 2010; 304 (20): 2253-62.

23. Earnest C, Johannsen N, Swift D, Gillison F, Mikus $\mathrm{C}$, et al. Aerobic and strength training in concomitant metabolic syndrome and type 2 diabetes. Med Sci Sports Exerc 2014; 46 (7): 1293-301.

24. Johannsen N, Sparks L, Zhang Z, Earnest C, Smith S, et al. Determinants of the changes in glycemic control with exercise training in type 2 diabetes: A randomized trial. PLoS ONE 2013; 8 (6): 1-6. Disponible en www.plosone. org [Consultado el 28 de julio de 2018]. 
25. Sénechal M, Swift D, Johannsen N, Blair S, Earnest C, et al. Changes in body fat distribution and fitness are associated with changes in hemoglobin Alc after 9 months of exercise training. Diabetes Care 2013; 36 (3): 2843-9.

26. Balducci S, Zanuso S, Cardelli P, Salvi L, Mazzitelli G, et al. Changes in physical fitness predict improvements in modifiable cardiovascular risk factors independently of body weight loss in subjects with type 2 diabetes participating in the Italian Diabetes and Exercise Study (IDES). Diabetes Care 2012; 35 (6): 1347-54.

27. Johansen M, MacDonald C, Hansen K, Karstoft K, Christensen $\mathrm{R}$, et al. Effect of an intensive lifestyle intervention on glycemic control in patients with type 2 diabetes. JAMA 2017; 318 (7): 637-46.

28. Larose J, Sigal R, Khandwala F, Prud'homme D, Boulé NG, et al. Associations between physical fitness and
HbA1c in type 2 diabetes mellitus. Diabetologia 2011; 54 (4): 93-102.

29. Plotnikoff R, Eves N, Jung N, Sigal R, Padwal R, et al. Multicomponent, home-based resistance training for obese adults with type 2 diabetes: a randomized controlled trial. Int J Obes 2010; 34 (6): 1733-41.

30. Prado P, Correa L, Alves A, Furlanetto R, Martins F, et al. Effect of resistance training on muscular strength and indicators of abdominal adiposity, metabolic risk, and inflammation in postmenopausal women: controlled and randomized clinical trial of efficacy of training volumen. AGE 2016; 40 (3): 1-13.

31. Segerstrom A, Glans F, Eriksson KF, Holmback A, Groop L, et al. Impact of exercise intensity and duration on insulin sensitivity in women with T2D. Eur J Int Med 2010; 21(6): 404-8. 